\title{
VERZEICHNIS DER LITERATURWISSENSCHAFTLICHEN HABILITATIONEN AN ÖSTERREICHISCHEN UNIVERSITÄTEN
}

\author{
Vorbemerkung der Redaktion
}

Ab 1985 bringen wir das ,Verzeichnis in jedem zweiten Halbband eines Jahrganges. Für diese Dokumentation laden wir neu Habilitierte ein, ihre Anzeige an die Redaktion 'Sprachkunst (Österreichische Akademie der Wissenschaften, Institut für Kulturwissenschaften und Theatergeschichte, Postgasse 7/4, IоIо Wien) jeweils bis spätestens Ende Juli zu schicken. Folgende Angaben werden erbeten: Name, Geburtsjahr, Beruf, Titel der Dissertation, Publikationsverzeichnis, Titel und Kurzfassung (ca 20 Zeilen/ı9o Wörter) der Habilitationsschrift, Venia, Institutszuordnung.

Priv.-Doz. Dr. Alexandra Strohmaier

Geboren: 1975

B e ru f: Priv.-Doz. am Institut für Germanistik der Universität Graz.

Dissertation:

Logos, Leib und Tod. Studien zur Prosa Friederike Mayröckers, Universität Graz 2004, 259 S. [Siehe Publikationen.]

Publikationen:

Monografien:

- Logos, Leib und Tod. Studien zur Prosa Friederike Mayröckers, München 2008.

- Poetischer Pragmatismus: Goethe und William James (= Textologie 6), Berlin 2019 [peer-reviewed, im Druck].

Herausgaben:

- Leopold von Sacher-Masoch, hrsg. gem. mit InGRId SpÖRK (= Dossier 20), Graz, Wien 2002.

- Veza Canetti, hrsg. gem. mit Ingrid Spörk (= Dossier 24), Graz, Wien 2005.

- Buchstabendelirien. Zur Literatur Friederike Mayröckers, Bielefeld 2009.

- Raum: Konzepte in den Künsten, Kultur- und Naturwissenschaften, hrsg. gem. mit Petra Ernst (= Stadt, Raum, Architektur. I), Baden-Baden 2013.

- Kultur - Wissen - Narration. Perspektiven transdisziplinärer Erzählforschung für die Kulturwissenschaften (= Kultur- und Medientheorie), Bielefeld 20 I3. 
Aufsätze:

- Auto(r)genesis. Zur Konstitution des masochistischen Subjekts im narrativen Akt, in: Leopold von Sacher-Masoch, hrsg. von Ingrid Spörk und Alexandra Strohmaier (= Dossier 20), Graz, Wien 2002, S. 90-I 20.

- Körper, Macht und Lust. Zur diskursiven Konstruktion des Masochismus, in: Phantom der Lust. Bd. I: Visionen des Masochismus. Essays und Texte, hrsg. von Peter Weibel, München 2003, S. I 50-160.

- Body, Power and Desire. On the Discursive Construction of Masochism, in: Phantom of Desire. Visions of Masochism. Essays and Texts. English Supplement, hrsg. von Peter WeibeL, München 2003, S. 85-90.

- Groteske Physiognomien. Zum semiotischen Konzept des Körpers in den Texten Veza Canettis, in: Veza Canetti, hrsg. von Ingrid Spörk und Alexandra Strohmaier (= Dossier 24), Graz, Wien 2005, S. I 2 I-I 47.

- Die Wirkmächtigkeit der Bilder. Zur (inter)medialen Konstruktion des hysterischen Körpers in Medizin, Kunst und Literatur, in: LiCuS. Journal of literary theory and cultural studies 2 (2007), S. I-I 8.

- „Barnow in Halb-Asien“. Zur narrativen Konstruktion des Raumes in den Texten von Karl Emil Franzos, in: Karl Emil Franzos - Schriftsteller zwischen den Kulturen, hrsg. von Petra Ernst (= Schriften des Centrums für Jüdische Studien I 2), Innsbruck u.a. 2007, S. I I-36.

- Diskurs, Macht, Raum. Grenzüberschreitende Zugänge zu kultureller Alterität am Beispiel eines habsburgischen Topos, in: Moderne. Kulturwissenschaftliches Jahrbuch 3 (2007): Themenschwerpunkt: Emotionen, hrsg. von Helga Mitterbauer und KathaRINA SCHERKe, S. 222-227.

- Zur Konstitution des Raumes durch diskursive und performative Praxis, in: Gedächtnis Identität - Differenz. Zur kulturellen Konstruktion des südosteuropäischen Raumes und ihrem deutschsprachigen Kontext, hrsg. von Marijan Bobinac und Wolfgang Müller-Funk (= Kultur - Herrschaft - Differenz I 2.), Tübingen, Basel 2008, S. 23-37.

- „Die Völker haben diesen Krieg wirklich nicht gewollt!“ Zur Narration des Großen Krieges in Alexander Granachs „Da geht ein Mensch“, in: transversal - Zeitschrift für Jüdische Studien 9 (2008), H. 2: Der Erste Weltkrieg, hrsg. von Petra Ernst, S. 7-24.

- Hybride Topographien. Galizien als Erinnerungsraum im autobiographischen Diskurs, in: Orte der Erinnerung. Kulturtopographische Studien zur Donaumonarchie, hrsg. von Eva Kocziszky (= Istros Bücher. Transnationale Studien zur vergleichenden Literaturund Kulturwissenschaft I), Budapest 2009, S. I 28-I 42.

- Der „Columbus des Ostens“. Leopold von Sacher-Masoch als literarischer Ethnograph der Habsburgermonarchie, in: Kunst und Wissenschaft aus Graz. Bd. 2: Kunst und Geisteswissenschaften aus Graz. Werk und Wirken überregional bedeutsamer Künstler und Gelehrter. Vom I 5 . Jahrhundert bis zur Jahrtausendwende, hrsg. von KarL AcHam, Wien u.a. 2009, S. 31 5-328.

- Prosa und/als Performanz. Zur performativen Ästhetik der Prosa Friederike Mayröckers, in: Buchstabendelirien. Zur Literatur Friederike Mayröckers, hrsg. von AleXandra Strohmaier, Bielefeld 2009, S. I 2 I-I 40.

- Zur Performativität des Narrativen. Vorüberlegungen zu einer performativen Narratologie, in: Language and World. Part Two. Signs, Minds and Actions, hrsg. von Volker Munz, Klaus Puhl und Joseph Wang (= Publications of the Austrian Ludwig Wittgenstein Society. New Series Volume I 5), Frankfurt/M. 2010, S. 77-93. 
- Kontingenz und Reflexion. Zur narrativen Poetik Sabine Grubers, in: Schreibweisen. Poetologien. Die (Post-)Moderne in der österreichischen Literatur von Frauen. Bd. 2, hrsg. von Hildegard Kernmayer, Wien 20 i i, S. 393-4I I.

- Masochismus, Macht und Monarchie. Szenarien der Unterwerfung in politischer und psychopathologischer Perspektive, in: Konfliktszenarien um I900. Politisch - sozial kulturell. Österreich-Ungarn und das Russische Imperium im Vergleich, hrsg. von Peter Deutschmann, Volker Munz und Olga Pavlenko, Wien 20 i i, S. 325-342.

- „Halb-Asiens“ umtriebige Körper. Zur Sexualisierung kultureller Alterität in der Habsburgermonarchie, in: Contested Passions. Sexuality, Eroticism, and Gender in Modern Austrian Literature and Culture, hrsg. von Clemens Ruthner und Raleigh WhitinGER (= Austrian Culture 46), New York u.a. 20 I I, S. 17-32.

- Zu Homi K. Bhabhas Theorem der kolonialen Mimikry, in: „Dritte Räume“. Homi K. Bhabhas Kulturtheorie. Kritik, Anwendung, Reflexion, hrsg. von Anna BabKa, Julia Malle und Matthias Schmidt, Wien 20i 2, S. 69-85.

- „Bekenntnisse haben nichts mit der Wahrheit zu tun“. Zur Performativität der Prosa Friederike Mayröckers. Punktuelle Anmerkungen, in: „Einzelteilchen aller Menschengehirne". Subjekt und Subjektivität in Friederike Mayröckers (Spät-)Werk, hrsg. von Françoise Lartillot, Aurélie Le Née und Alfred Pfabigan, Bielefeld 20 i 2, S. i i 5-I 30.

- Für einen performativitätstheoretischen Zugang zur autobiographischen Konstruktion Galiziens am Beispiel der Lebenserzählungen Alexander Granachs und Ella Schapiras, in: Exklusion, Inklusion, Repräsentation: Galizien im Diskurs, hrsg. von PAula Giersch, Florian Krobb und Franziska Schössler (= Inklusion/Exklusion. Studien zu Fremdheit und Armut von der Antike bis zur Gegenwart), Frankfurt/M. 20 I2, S. $225-242$.

- Entwurf zu einer performativitätstheoretischen Narratologie am Beispiel der Rahmenzyklen Goethes, in: Kultur - Wissen - Narration. Perspektiven transdisziplinärer Erzählforschung für die Kulturwissenschaften, hrsg. von Alexandra Strohmaier (= Kultur- und Medientheorie), Bielefeld 2013, S. I99-23I.

- Relationale und mediatisierte Räume. Zu einer Raumkonzeption Goethes und deren Aktualität, in: Raum. Konzepte in den Künsten, Kultur- und Naturwissenschaften, hrsg. von Petra Ernst und Alexandra Strohmaier (= Stadt, Raum, Architektur i), BadenBaden 2013, S. I 57-183.

- Annäherungen an eine kulturwissenschaftliche Narratologie des Raumes am Beispiel von Goethes „Novelle“, in: Topographie und Raum in der deutschen Sprache und Literatur, hrsg. von Fabrizio Cambi und Wolfgang Hackl (= Stimulus. Mitteilungen der Österreichischen Gesellschaft für Germanistik), Wien 20 I3, S. 40-63.

\section{Rezensionen:}

- Friederike Mayröcker, Und ich schüttelte einen Liebling, Frankfurt/M. 2005, in: kolik. Zeitschrift für Literatur 32 (2005), S. I62-165.

- Christina Lechtermann, Kirsten Wagner, Horst Wenzel (Hrsgg.), Möglichkeitsräume. Zur Performativität sensorischer Wahrnehmung, Berlin 2007, in: Moderne. Kulturwissenschaftliches Jahrbuch 4 (2008): Themenschwerpunkt: Migration, hrsg. von Helga Mitterbauer und Katharina Scherke, S. $276 f$.

- Inge Arteel, gefaltet, entfaltet. Strategien der Subjektwerdung in Friederike Mayröckers Prosa 1988-1998, Bielefeld 2007, in: Germanistische Mitteilungen, H. 70 (2009), S. $77 f$. 
Habilitationsschrift:

Poetischer Pragmatismus. Goethe und William James, Universität Graz 2017, 334 S. [Siehe Publikationen.]

Kurzfassung:

Die Habilitationsschrift zeigt erstmals die historischen und systematischen Beziehungen zwischen den Werken Goethes und William James' auf. Dabei wird zum einen, unter Einbezug von Archivmaterial, die Goethe-Lektüre des US-amerikanischen Philosophen in ihrer Bedeutung für die Genese und Poetizität des James'schen Pragmatismus rekonstruiert. Zum anderen wird die (proto-)pragmatische Dimension von Goethes Werk herausgearbeitet und dieser als literarischer Philosoph und Wegbereiter des angloamerikanischen Pragmatismus exponiert. Mit der Konturierung der vielfältigen diskursiven Relationen zwischen literarischen, philosophischen und naturwissenschaftlichen Wissensordnungen der Goethezeit und jenen der US-amerikanischen Kultur des späten I9. und beginnenden 20. Jahrhunderts gewinnt der Pragmatismus als transatlantische Denkbewegung Profil. Auf theoretischmethodischer Ebene leistet die Arbeit einen maßgeblichen Beitrag zum interdisziplinären Forschungsfeld „Literatur und Philosophie“, wobei neben der Rolle literarisch verfasster Philosopheme insbesondere auch das epistemische Potential literarischer Verfahren und deren spezifische Funktionen für die Konstitution philosophischer Diskurse und Praktiken demonstriert werden.

Venia:

Neuere deutschsprachige Literatur und Allgemeine und Vergleichende Literaturwissenschaft.

Institutszuordnung:

Institut für Germanistik der Universität Graz.

Priv. Doz. Mag. Dr. Daniela Strigl

Geboren: 1964

B eruf: Literaturkritikerin, Lehrbeauftragte am Institut für Germanistik der Universität Wien.

Dissertation:

Theodor Kramer - Heimatdichter und Sozialdemokrat zwischen den Fronten. Ein Beitrag zur österreichischen Lyrik der Zwischenkriegszeit, Wien I99I, 345 S.. [Siehe Publikationen.]

Publikationen:

\section{Monografien:}

- „Wo niemand zuhaus ist, dort bin ich zuhaus“. Theodor Kramer - Heimatdichter und Sozialdemokrat zwischen den Fronten (= Literatur in der Geschichte, Geschichte in der Literatur Bd. 25), Wien-Köln-Weimar 1993. 
- Marlen Haushofer. Die Biographie, München 2000.

- „Wahrscheinlich bin ich verrückt ..." Marlen Haushofer - die Biographie, München 2009.

- „Berühmtsein ist nichts“. Marie von Ebner-Eschenbach. Eine Biographie, Wien, Salzburg 2016.

- Alles muss man selber machen. Biographie. Kritik. Essay, Graz 2018.

\section{Herausgaben:}

- Walter Buchebner, zeit aus zellulose. Gedichte. Mit einem Nachwort von Daniela Strigl, Graz I994.

- Frauen verstehen keinen Spaß (= Profile/Magazin des österreichischen Literaturarchivs 9), Wien 2002.

- (Zus. mit Friedbert Aspetsberger:) „Ich kannte den Mörder, wußte nur nicht, wer er war." Zum österreichischen Kriminalroman der Gegenwart (= Schriftenreihe Literatur des Instituts für Österreichkunde Bd. I4), Innsbruck u.a. 2004.

- (Zus. mit Erwin Chvojka, Erich Hackl u.a.:)Theodor Kramer, Solange der Atem uns trägt. Gedichte. Wien 2004.

- Francisco Tanzer, Der Österreicher in mir. Leben und Werk, Wien 2006.

- (Zus. mit Evelyne Polt-Heinzl:) Im Keller. Der Untergrund des literarischen Aufbruchs nach I945, Wien 2006.

- (Zus. mit Miguel Herz-Kestranek, Konstantin Kaiser:) In welcher Sprache träumen Sie? Österreichische Exillyrik, Wien 2007.

- (Zus. mit Herbert Ohrlinger:) Grenzgänge. Der Schriftsteller Karl-Markus Gauß, Wien 2010.

- (Zus. mit Stefan Krammer:) Österreichische Gegenwartsliteratur 2000-20io. ide 4/20II.

- Walter Buchebner, ich die eule von wien. Gedichte, Manifeste, Tagebücher, Wien, 2. erw. Aufl. 2012.

- (Zus. mit Stephan Kurz, Michael Rohrwasser:) Der Dichter und sein Germanist. In Memoriam Wendelin Schmidt-Dengler, Wien 20 I 2.

- (Zus. mit Klaus Kastberger:) Richard Billinger - Heimat. Körper. Kunst. Richard Billinger Symposium (= Schriften zur Literatur und Sprache in Oberösterreich 20), Linz 2014 .

- (Zus. mit Evelyne Polt-Heinzl, Ulrike Tanzer:) Marie von Ebner-Eschenbach, Leseausgabe in vier Bänden. Mit Vorwort und Kommentar. Wien, Salzburg 20 I $4 f f$.

- (Zus. mit Roland Innerhofer:) Sonderweg in Schwarzgelb? Auf der Suche nach einem österreichischen Naturalismus in der Literatur, Innsbruck 2016.

- (Zus. mit Karl Wagner:) Peter Rosegger, Ausgewählte Werke in Einzelbänden. [4 Bde.] Mit Materialien, Kommentar und Nachwort, Wien 2018.

Aufsätze

- Zwischen Hölderlin und Kerouac. Walter Buchebners Via dolorosa zur „Activen Poesie“, in: Zagreber Germanistische Beiträge H. 3 (I996), S. 73-84.

- Literarische Amtshandlungen. Albert Drachs Protokolle, in: 50 Jahre 2. Republik - I000 Jahre „Ostarrichi“. Germanistische Mitteilungen (Brüssel) H. 43-44 (I996) , S. 69-80.

- Versuch über Wilhelm Szabo, in: Literatur und Kritik, H. 3 I7-3 I 8 (I997), S. 48-55. 
- Zwei Fliegen auf einen Schlag. Eine Theodor-Kramer-Parodie Peter Hammerschlags, in: Kringel, Schlingel, Borgia. Materialien zu Peter Hammerschlag, hrsg. von MoniKa Kiegler-Griensteidl und Volker Kaukoreit, Wien i 997, S. I95-206.

- Worüber kein Gras wächst. Hans Leberts politische Lektion, in: Hans Lebert, hrsg. von Gerhard Fuchs und Günther A. Höfler (= Dossier I2), Graz, Wien I997, S. II7-I42.

- Theodor Kramer, „Der Wurf am Kai“, in: Der literarische Einfall. Über das Entstehen von Texten. Profile i/r998, hrsg. von Bernhard Fetz und Klaus Kastberger, Wien I998, S. I66-I76.

- In der Reißmaschine der Zeit. Zur hautnahen Vergangenheitsbewältigung in Andreas Okopenkos „Kindernazi“, in: Andreas Okopenko. Texte und Materialien, hrsg. von Klaus Kastberger, Wien i998, S. i03-I20.

- Spurensicherung auf dem „österreichischen NS-Parnaß“. Otto Basil und die Debatte um Josef Weinheber, in: Otto Basil und die Literatur um 1945. Profile 2/1998, hrsg. von Volker Kaukoreit und Wendelin Schmidt-Dengler, Wien i998, S. 66-76.

- Vertreibung aus dem Paradies. Marlen Haushofers Existentialismus, in: Anke Bosse, Clemens Ruthner (Hrsgg.), „Eine geheime Schrift aus diesem Splitterwerk enträtseln ..."Marlen Haushofers Werk im Kontext, Tübingen-Basel 2000, S. I 2 I-I 36.

- „Erschrocken fühl ich heut mich dir verwandt“. Theodor Kramer und Josef Weinheber, in: Chronist seiner Zeit - Theodor Kramer, hrsg. von Herbert Staud und Jörg ThunECKE (= Zwischenwelt 7), Klagenfurt 2000, S. 255-274.

- Winterglück und -unglück. Zur Alterslyrik Friederike Mayröckers, Ernst Jandls, Gerald Bisingers und Michael Guttenbrunners, in: Die Lebenden und die Toten. Beiträge zur österreichischen Gegenwartsliteratur, hrsg. von Markus Knöfler, Peter Plener und Péter Zalán (= Budapester Beiträge zur Germanistik 35), Budapest 2000, S. 4I-56.

- Melancholie im Spätkapitalismus, in: Lynkeus 2 (2000), hrsg. von Christian SchacherREITER im Auftrag des Adalbert-Stifter-Institutes des Landes Oberösterreich, S. 73-76.

- Kunst- und Ruinenblumen. Kaffeehaus und literarische Geselligkeit im NachkriegsWien, in: „Displaced“. Paul Celan in Wien I 947-I948, hrsg. von Peter Gossens und Marcus G. Patka, Frankfurt/M. 200I, S. 89-98.

- Fräulein- und andere Wunder. Galvagni, Röggla \& Co, in: Geschlechter. Essays zur Gegenwartsliteratur, hrsg. von Friedbert Aspetsberger und Konstanze Fliedl (= Schriftenreihe Literatur des Instituts für Österreichkunde), Innsbruck u.a. 200I, S. I3 I-I 54 .

- „Die Lebenden leben und die Toten sind tot“. Norbert Gstreins „Die englischen Jahre“, in: Wieland Freund, Winfried Freund (Hrsgg.), Der deutsche Roman der Gegenwart. München 200I, S. 224-229.

- Die im Dunkeln und die im Licht. Was einer freischaffenden Rezensentin an bedeutenden Gegenwartsautor/inn/en auffällt, in: Ein Dichter-Kanon für die Gegenwart, hrsg. von Friedbert Aspetsberger, Innsbruck u.a. 2002, S. I03-I 27.

- Minimalismus als Anspruch. Über das Kulinarische in Elfriede Gerstls Lyrik, in: Elfriede Gerstl, hrsg. von Konstanze Fliedl und Christa Gürtler, Graz, Wien 2002, S. $165-185$.

- Lauter Fälle - nicht nur für die Literaturwissenschaft. Über den Hang zum Medizinischen zum Beispiel bei Paulus Hochgatterer, Melitta Breznik und Thomas Raab, in: (Nichts) Neues. Trends und Motive in der (österreichischen) Gegenwartsliteratur, hrsg. von Friedbert Aspetsberger, Innsbruck u.a. 2003, S. I 58-I79. 
- „niemand bespricht unsern Staub“. Paul Celans Strategien des Scheiterns. Vortrag bei: Austrian Writers Confront the Past 1945-2000. An International Conference at the University of Pennsylvania, Philadelphia, April 2002, in: New German Critique Nr. 93 (2004), hrsg. von Stephen Beller und Frank Trommler, S. $63-72$

- „Die Wand” (1963) - Marlen Haushofers Apokalypse der Wirtschaftswunderwelt. Vortrag bei: Das Verbindende der Kulturen, INST Konferenz, Konferenzzentrum Wien, November 2003. <http://www.inst.at/trans/ I $5 \mathrm{Nr} / 0$ 5_I6/strigl I 5.htm>

- Das unfaßbare Genie. Zu aktuellen Künstlerromanen von Hans-Ulrich Treichel und Daniel Kehlmann, in: Kunst und Musik in der Literatur. Ästhetische Wechselbeziehungen in der österreichischen Literatur der Gegenwart. II. bilaterales germanistisches Symposion, Brünn, Tschechien, Dezember 2003, hrsg. von Roman Kopřiva und JaroslaV Kovář, Wien 2004, S. IOI-I I 8.

- Warum sie so gute Bücher schreiben. Denker als Dichter, in: Klaus Kastberger, Konrad Paul Liessmann (Hrsgg.), Die Dichter und das Denken. Wechselspiele zwischen Literatur und Philosophie (= Profile I I), Wien 2004, S. 7-3 I.

- Der Hedonismus und der Tod. Warum in Krimis so viel gegessen und getrunken wird, in: „Ich kannte den Mörder, wußte nur nicht, wer er war.“ Zum österreichischen Kriminalroman der Gegenwart, hrsg. von Friedbert Aspetsberger und Daniela Strigl, Innsbruck 2004, S. I 2 I-I 43.

- Theodor Kramer (I897-1958). Heimatdichter, Jude, Sozialist, in: Hinter den Bergen ist eine andere Welt. Österreichische Literatur des 20. Jahrhunderts, hrsg. von Anke Bosse und Leopold Decloedt (= Duitse Kroniek 53), Amsterdam, New York 2004, S. I37-I 59.

- „Es gibt Taten, die so ungeheuer sind, daß keine Sühne hilft“. Über das Zeitgemäße an Lernets "Germanien“ (I946), in: HélÈne Barrière, Thomas Eicher und Manfred MülLER (Hrsgg.), Schuld-Komplexe. Das Werk Alexander Lernet-Holenias im Nachkriegskontext (= Übergänge Grenzfälle. Österreichische Literatur in Kontexten Io), Oberhausen 2004, S. 65-89.

- Unter die Haut. Lebens- und Todesarten im „spätbürgerlichen“ Zeitalter: Haushofer, Bachmann, Jelinek, in: Leiden ... Genießen. Zu Lebensformen und -kulissen in der Gegenwartsliteratur, hrsg. von Friedbert Aspetsberger und Gerda A. Moser, Innsbruck 2005, S. I39-163.

- Mit dem Hammer dichten. Zur Alterslyrik Ernst Jandls, in: Ernst Jandl. Musik Rhythmus Radikale Dichtung. Profile r $2 / 2005$, hrsg. von Bernhard Fetz, Wien 2005, S. 53-7I.

- Geher und Untergeher. Zur tödlichen Subordination unter das Genie, in: UnterOrdnungen - Herrschaft, (Ohn)Macht und Anarchie bei Thomas Bernhard, hrsg. von Clemens Ruthner und Sonja Malzner. Germanistische Mitteilungen 60-6I/2004-2005 (= Acta Austriaca-Belgica 4), S. 6I-74.

- Das unfaßbare Genie. Zu aktuellen Künstlerromanen von Hans-Ulrich Treichel und Daniel Kehlmann, in: Kunst und Musik in der Literatur. Ästhetische Wechselbeziehungen in der österreichischen Literatur der Gegenwart, hrsg. von Roman Kopriva und Jaroslav Kovar, Wien 2005, S. IOI-Ii 8.

- „Die Partei der Armut und der Freiheit“ - Guttenbrunners Griechenland, in: Michael Guttenbrunner. Texte und Materialien, hrsg. von Manfred Müller und Helmuth Niederle, Wien 2005, S. 49-68.

- Schneidige Husaren, brave Bosniaken, feige Tschechen - nationale Mythen und Stereotypen in der k.u.k. Armee, in: Zentren, Peripherien und kollektive Identitäten in 
Österreich-Ungarn, hrsg. von Endre Hárs u.a. (= Kultur - Herrschaft - Differenz 9), Tübingen, Basel 2006, S. I29-I43.

- „stadt im fetten walfischbauch“. Wien-Bilder in der zeitgenössischen Literatur, in: Imaging Vienna. Innensichten, Außensichten, Stadterzählungen, hrsg. von MonIKA Sommer, Marcus Gräser, Ursula Prutsch, Wien 2006, S. I22-I 37.

- „Ich bin der Staub, mit dem man Kehraus macht." Theodor Kramers Altersgedichte zwischen Reminiszenz, barocker Klage und Drastik, in: Echo des Exils. Das Werk emigrierter österreichischer Schriftsteller nach I945, hrsg. von Jörg THunecke, Wuppertal 2006, S. I IO-I 22.

- Walter Buchebner - Dichter unter Hochdruck, in: Im Keller. Der Untergrund des literarischen Aufbruchs nach 1945, hrsg. von Evelyne Polt-Heinzl und Daniela Strigl, Wien 2006, S. 26-35.

- „Zu jedem Erfolg gehören fünfzig Prozent Dummheit.“ Albert Drachs „Unsentimentale Reise“ und Soma Morgensterns „Flucht in Frankreich“, in: Diaspora - Exil als Krisenerfahrung. Jüdische Bilanzen und Perspektiven, hrsg. von Armin Eidherr, Gerhard Langer und Karl Müller (= Zwischenwelt io), Klagenfurt 2006, S. 378-393.

- Gegen die „Wand“. Zu Elfriede Jelineks Lektüre von Marlen Haushofers Roman in „Der Tod und das Mädchen V“, in: Modern Austrian Literature, Nr. 39 (2006), S. 73-96.

- ,Fremdheiten'. Österreichische Lyrik der Zwischenkriegszeit. Jakob Haringer, Theodor Kramer, Wilhelm Szabo, Guido Zernatto, in: Literatur und Kultur im Österreich der Zwanziger Jahre. Vorschläge zu einem transdisziplinären Epochenprofil, Bielefeld 2007, S. I79-I94.

- „Das Fräulein Doctor ist eine langsame, aber ausgezeichnete Arbeiterin.“ Bachmanns „kritische" Schriften und ihr öffentliches Bild, in: Ingeborg Bachmann. Neue Bilder zu ihrer Figur, hrsg. von Friedbert Aspetsberger, Innsbruck 2007, S. I77-20 I.

- Was den Reisenden bewegt. Über Fernweh, Heimweh, Utopie und Nostalgie, in: In die weite Welt hinein. Reisen in der Kinder- und Jugendliteratur, hrsg. vom Institut für Jugendliteratur, Wien 2007, S. 4-I 2.

- Seher, Emphatiker, Gnostiker. Literaturkritik und Literaturtheorie, in: Germanistik und Literaturkritik. Zwischenbericht zu einer wunderbaren Freundschaft, hrsg. von PRIMUsHeinz Kucher und Doris Moser (= Stimulus/Mitteilungen der Österreichischen Gesellschaft für Germanistik 2006), Wien 2007, S. 35-48.

- Brigitte Schwaiger, „Wie kommt das Salz ins Meer“ (I 977). Rückblick auf ein „Fräuleinwunder" - cum grano salis, in: Grundbücher der österreichischen Literatur seit 1945. Erste Lieferung. Profile I 4/2007, hrsg. von Klaus Kastberger und Kurt Neumann unter Mitarbeit von Michael Hansel, Wien 2007, S. 249-258.

- Leicht wie ein Vogel - Elfriede Gerstl, in: Falter, I3.6.2007.

- Literary Perspectives: Austria. Alles andere als ein deutscher Wurmfortsatz, in: Eurozine. $<$ http://www.eurozine.com/articles/2008-o6-Io-strigl-en.html>

- Neue Wege, alte Schuhe. Theodor Kramer als Autor der klassischen Moderne, in: Volltext 5/2008. S. 8-10

- Giftkorn der Wut. Über den Polemiker Walther Rode, in: Volltext 6/2008. S. rof.

- Heißt lachen. Einiges zur Komik im Werk Margit Schreiners, in: Die Rampe 3/2008, S. $5 \mathrm{I}-55$.

- Ihr Auftritt, bitte! Sprachingenieure als Entertainer, in: Thomas Eder, Juliane Vogel (Hrsgg.), verschiedene sätze treten auf. Die Wiener Gruppe in Aktion (= Profile I5), Wien 2008, S. 9-28. 
- Hölderlin galvanisiert. Zum Verhältnis von Ironie und Pathos bei Christian Loidl, in: Leopold Federmair, Helmut Neundlinger (Hrsgg.), Christian Loidl (I957-200i). Beiträge zu Leben und Werk des Dichters, Linz 2008, S. I22-I 39.

- Stein, Rinde, Blatt. Zu Christines Bustas Theologie des Staunens, in: Christine Busta. Texte und Materialien, hrsg. von Michael Hansel, Wien 2008, S. 29-43.

- Die „Neuen Wege“-Zentralorgan der Avantgarde? in: Gerald M. Bauer, Birgit Peter (Hrsgg.), „Neue Wege“. 75 Jahre Theater der Jugend in Wien, Wien 2008, S. 73-86.

- „Ich seh, ich seh, was du nicht siehst“ - Literaturkritik und Literaturtheorie, in: Derrida und danach? Literaturtheoretische Diskurse der Gegenwart, hrsg. von Georg ThusWALDNER, Wiesbaden 2008, S. I 45-I 53.

- Anspruchsvolle Armut? Zur Lyrik von Theodor Kramer und Guido Zernatto, in: Elke Brüns (Hrsg.), Ökonomien der Armut. Soziale Verhältnisse in der Literatur, München 2008, S. I73-I 88.

- Der kriegerische Pazifist. Walther Rode und das Jahr i 91 8, in: 8ung, hrsg. von Gerfried Sperl und Michael Steiner (= Was für Zeiten io), Graz 2008, S. 47-55.

- Vom Rasen (Furor). Ein Versuch zu Friederike Mayröckers Affektpoetik, in: Buchstabendelirien. Zur Literatur Friederike Mayröckers, hrsg. von AleXAndra Strohmaier, Bielefeld 2009, S. 5I-73.

- Die Seelen der Toten, die „toten Seelen der Lebenden“ und das „Klitterungsklistier“ des Herr Geschichtsprofessors. Elfriede Jelinek und die Nachgeborenen, in: Pia Janke, Teresa Kovacs, Christian Schenkermayr (Hrsgg.), Die endlose Unschuldigkeit“. Elfriede Jelineks „Rechnitz (Der Würgeengel), Wien 20 I0, S. 36I-376.

- Der Biograph als Testamentsvollstrecker. Anton Bettelheim erfindet Marie von EbnerEschenbach, in: Stephan Kurz, Michael Rohrwasser, Daniela Strigl (Hrsgg.), Der Dichter und sein Germanist. In Memoriam Wendelin Schmidt-Dengler (= Zur neueren Literatur Österreichs 26), Wien 20 I 2, S. I I 2-1 30.

- Biographie als Intervention. Zum Problem biographischen Erzählens bei Stefan Zweig - Fouché und Erasmus, in: Karl Müller (Hrsg.), Stefan Zweig - Neue Forschung (= Schriftenreihe des Stefan Zweig Centre Salzburg 3), Würzburg 20 I 2, S. 9-25.

- Kling in Wien. Zu einem literarischen Myzel, in: Das Gellen der Tinte. Zum Werk Thomas Klings, hrsg. von Frieder von Ammon, Peer Trilcke, Alena Scharfschwert (= Deutschsprachige Gegenwartsliteratur und Medien 9), Göttingen 20 I 2, S. 8 I-I I 2.

- Der Kritiker. Gatekeeper, Platzanweiser, Zirkulationsagent, Raumpfleger oder Verkehrspolizist? Über die Literatur als herrschaftsfreie Zone, in: (Ver)Führungen. Räume der Literaturvermittlung, hrsg. von Meri Disoski, Ursula Klingenböck, Stefan Krammer, Innsbruck u.a. 201 2, S. 43-55.

- Christine Nöstlinger, „Iba de gaunz oaman kinda“ (I974), in: Grundbücher der österreichischen Literatur seit 1945. Zweite Lieferung. Profile 20/2013, hrsg. von KLaus Kastberger und Kurt Neumann, Wien 2013, S. i65-172.

- Diesseits der „Wand“ - Schreckensort oder Utopie? Die fabelhafte Welt der Marlen Haushofer, in: Grundbücher der österreichischen Literatur seit I945. Zweite Lieferung. Profile 20/20I3, hrsg. von Klaus Kastberger und Kurt Neumann, Wien 2013, S. 207-2I 4.

- Parforceritt in die Moderne. Hugo von Hofmannsthals „Reitergeschichte“, in: VioletTA L. Waibel, Konrad Paul Liessmann (Hrsgg.), Es gibt Kunstwerke - Wie sind sie möglich? Paderborn 2014, S. 333-352.

- „Ich war Kraus-geweiht noch ehe ich ein Wort von ihm kannte.“ Elazar Benyoëtz und 
Karl Kraus, in: Elazar Benyoëtz. Korrespondenzen. Profile 2I/20I4, hrsg. von BerNhard Fetz, Michael Hansel und Gerhard Langer, Wien 2014, S. i92-207.

- Boden ohne Blut? Billingers Lyrik und die Dresdner Zeitschrift „Die Kolonne“, in: Richard Billinger - Heimat. Körper. Kunst. Richard Billinger Symposium, hrsg. von KLAus Kastberger, Daniela Strigl (= Schriften zur Literatur und Sprache in Oberösterreich 20), Linz 20I4, S. I7-28.

- „I want a hero“ - Stefan Zweig und Lord Byron, in: Zweigs England, hrsg. von RüDIGer Görner und Klemens Renoldner (= Schriftenreihe des Stefan Zweig Centre Salzburg 5), Würzburg 20I4, S. 35-48.

- Jenseits von Krambambuli. Die phallischen Anmaßungen der Schriftstellerin Marie von Ebner-Eschenbach, in: Volltext 2/20I 5. I, S. 28-3 I.

- Die Geschichte umschreiben. Milo Dors Roman „Der letzte Sonntag“/,Die Schüsse von Sarajewo“, in: Vahidin Preljevic, Clemens Ruthner (Hrsgg.), „The Long Shots of Sarajevo" I 9I 4. Ereignis - Narrativ - Gedächtnis (= Kultur - Herrschaft - Differenz 22), Tübingen 2016, S. 505-516.

- „Mich kann man nicht verurteilen“ - Naturrecht und k. k. Justiz bei Marie von Ebner-Eschenbach und Ferdinand von Saar, in: Roland Innerhofer, Daniela Strigl (Hrsgg.), Sonderweg in Schwarzgelb? Auf der Suche nach einem österreichischen Naturalismus in der Literatur, Innsbruck 20 I6, S. I I I-I 25.

- „Und die Größe ist gefährlich.“ Über den schwierigen Umgang mit einem Klassiker, in: Franz Grillparzer. Ein Klassiker für die Gegenwart. Profile 23/20 i6, hrsg. von Bernhard Fetz, Michael Hansel und Hannes Schweiger, Wien 20i6, S. 9-23.

- Stefan Zweig und das Odol-Prinzip. Vom Erfinden einer Marke, in: Clemens Peck, Norbert Christian Wolf (Hrsgg.), Poetologien des Posturalen. Autorschaftsinszenierungen in der Literatur der Zwischenkriegszeit, Paderborn 2017, S. 26I-277.

- Von Teddybären, dem morbus austriacus und der Neugier auf die Welt. Gauß und die österreichische Literatur seiner Generation, in: Von der Produktivkraft des Eigensinns. Die Literaturen des Karl-Markus Gauß, hrsg. von Werner Michler, Klemens Renoldner und Norbert Christian Wolf, Salzburg 2017, S. i 5-29.

- Zwischen de Sade und Sacher-Masoch. Einschlägiges aus der Literatur, in: texte. psychoanalyse. ästhetik. kulturkritik H. 2/2017, Sadomasochismus in Klinik und Kultur, S. 89-107.

- Zwischen Stifter und Schnitzler. Marie von Ebner-Eschenbach - Zeitgenossin der Moderne, in: Stifter Jahrbuch Neue Folge 3I (2017), S. 9I-I07.

- I9I8 - das Ende einer "Justamentposition gegen die Natur" (Walther Rode). Über den Mythos vom „habsburgischen Mythos“ in der Literatur, in: Peter Becher, Florian KüHrer-Wielach (Hrsgg.) ... Zwischen Trauer und Triumph. Das Jahr I9I 8 in der mitteleuropäischen Literatur, Regensburg 2018, S. I7-38.

- Der „universalste Dilettant“ als „Baumeister der Welt“ - Zu Zweigs biographischem Essay über Giacomo Casanova, in: Arturo Larcati, Klemens Renoldner (Hrsgg.), „Am liebsten wäre mir Rom!“ Stefan Zweig und Italien (= Schriftenreihe des Stefan Zweig Zentrum Salzburg 9), Würzburg 201 8, S. 99-1 I7.

- „Ich bin so eine Reitnärrinn, daß ich vor dem Aufsteigen am ganzen Leibe zittere.“ Marie von Ebner-Eschenbachs Träume vom Reiten, in: Maria Piok, Ulrike Tanzer, Kyra Waldner (Hrsgg.), Marie von Ebner-Eschenbach. Schriftstellerin zwischen den Welten (= Innsbrucker Beiträge zur Kulturwissenschaft Germanistische Reihe 90), Innsbruck 2018, S. 4I-56. 
- Zwischen Morbus und Mythos. Kaiser Franz Joseph und das Habsburgische in den Werken des Exils, in: Elisabeth Erdem, Juliana P. Perez, Pedro Heliodoro de Moraes Branco Tavares (Hrsgg.) ... Stefan Zweig - Das Exilprojekt (= Schriftenreihe des Stefan Zweig Zentrum Salzburg Io), Würzburg 20 I9 [in Druck].

- „Wir lieben einander wie Mohn und Gedächtnis“. Bachmann und Celan und das Gedicht nach Auschwitz, in: Violetta Waibel (Hrsg.), Liebe und Hass in Philosophie, Religion und Literatur, Paderborn 2019 [in Druck].

\section{Lexikonartikel, Handbücher- und Katalogtexte:}

- Mitarbeit am Katalog der Ausstellung „Displaced’. Paul Celan in Wien 1947-1948“ im Jüdischen Museum Wien, Frankfurt/M. 200I.

- Michael Guttenbrunner, in: Kritisches Lexikon zur deutschsprachigen Gegenwartsliteratur, hrsg. von Heinz-Ludwig ARnold, München (67. NL März 200I).

- Marlen Haushofer, in: Killy Literaturlexikon. 2. Auf, hrsg. von Wilhelm KühlmanN u.a. Berlin 2008.

- Artikel zu Karl-Markus Gauß, in: Kritisches Lexikon zur Gegenwartsliteratur, hrsg. von Heinz Ludwig Arnold, io. Nachlieferung 2009.

- Pia Janke (Hrsg.), Jelinek-Handbuch. Stuttgart, Weimar: Metzler 2013 [Beitrag zu "Neid"].

- Schach und andere Leidenschaften oder Stefan Zweigs Liebe zur Niederlage, in: Stefan Zweig - Abschied von Europa, hrsg. von Klemens Renoldner, Wien 20i4, S. I23I 35 .

- Marion Bönnighausen, Jochen Vogt (Hrsgg.), Literatur für die Schule. Ein Werklexikon für den Deutschunterricht, Paderborn 2014 [Artikel zu Ebner-Eschenbach: Das Gemeindekind].

- Arturo Larcati, Klemens Renoldner, Martina Wörgötter (Hrsgg.), Stefan Zweig Handbuch, Berlin 2018 [Beiträge zu „Joseph Fouché“ und „Marie Antoinette“].

- „Frauenverehrer“, „Liebessklave“, „Gott und Teufel“ - zu Karl Kraus“ erotischer Biographie, in: Katharina Prager (Hrsg.), Geist versus Zeitgeist. Karl Kraus in der Ersten Republik. [Katalog] Wien 2018, S. I66-18I.

Habilitationsschrift:

Prolegomena zu einer neuen Biographie Marie von Ebner-Eschenbachs. „Berühmtsein ist nichts." Marie von Ebner-Eschenbach. Eine Biographie, Wien 2018.

Kurzfassung:

Die Habilitationsschrift besteht im Hauptteil aus der 20 I 6 im Residenz Verlag publizierten Biographie der österreichischen Schriftstellerin Marie von Ebner-Eschenbach (1830-1916) sowie aus einer theoretischen und forschungsgeschichtlichen Einleitung, die mit der Methodenreflexion ein Bekenntnis zum Prinzip der biographischen Narration wie zu Diltheys Forderung ablegt, das Individuum als „Kreuzungspunkt für Kultursysteme“ zu betrachten. Die Biographie füllt eine Forschungslücke, die sich seit Anton Bettelheims Werk „Marie von Ebner-Eschenbach. Wirken und Vermächtnis“ (I920) aufgetan hat. Sie setzt der verklärendverehrenden Haltung des Freundes eine Position kritisch-distanzierter Würdigung entgegen, die einerseits der Ästhetik von Ebner-Eschenbachs heute vielfach unterschätzter Literatur gilt, andererseits ihrer für eine Aristokratin bemerkenswert fortschrittlichen politischen Anschauung, in der die Diskurse der franzisko-josephinischen Epoche evident werden. Dem 
gängigen Bild einer Dichterin des Mitleids und der Güte werden dezidiert andere Facetten entgegengehalten: die - gerade gegenüber dem eigenen Stand - scharfsichtige Sozialkritikerin, die „Emanzipierte“, die Satirikerin, die Liberale und Antiklerikale. Ausgehend vom Status einer breitenwirksamen Kultautorin um I900, verfolgt die biographische Erzählung den Weg eines von unbeirrbarem künstlerischen Ehrgeiz getragenen Talents: von den Widerständen der gräflichen Herkunftsfamilie Dubsky und des Cousins und späteren Gatten Moriz von Ebner-Eschenbach, über die ersten kleinen Erfolge und großen Niederlagen als Dramatikerin und die Profilierung als Aphoristikerin und Prosaautorin im fortgeschrittenen Alter bis zum durch Selbststilisierung mitgestalteten Bild der Berühmten.

Venia:

Neuere deutsche Literatur.

Institutszuordnung:

Institut für Germanistik der Universität Wien.

Assoz. Prof. Dr. Silvia Schultermandl

Geboren: 1977

Beruf: Assoziierte Professorin am Institut für Amerikanistik an der Karl-Franzens-Univeristät Graz.

Dissertation:

Unlinear Matrilineage: Mother-Daughter Relationships and the Politics of Location in Asian American and Caribbean American Literature, Graz 2004, 235 S.

Publikationen:

\section{Monografien:}

- Transnationalism as Aesthetic Experience: States of Ambivalence from Equiano to 9/I I. Forthcoming.

- Transnational Matrilineage: Mother-Daughter Conflicts in Asian American Literature. (= Contributions to Transnational Feminism I) Vienna 2009.

\section{Herausgaben:}

- (Zus. mit May Friedman:) Autobiography 2.0 and Quick Media Life Writing. Special issue of Interactions: Studies in Communications and Culture 9.2 (2018).

- (Zus. mit May Friedman:) Click and Kin: Transnational Identity and Quick Media, Toronto 2016.

- (Zus. mit Petra Eckhard und Klaus Rieser:) Localizing Global Phenomena: The Contact Spaces of American Culture (= American Studies in Austria Io), Vienna 2012.

- (Zus. mit May Friedman:) Growing Up Transnational: Identity and Kinship in a Global Era, Toronto $20 \mathrm{II}$.

- (Zus. mit Sebnem Toplu:) A Fluid Sense of Self: The Politics of Transnational Identity in Anglophone Literatures (= Contributions to Transnational Feminism 2), Vienna 20 Io.

- Against the (Main)Stream: New Perspectives on Asian American and Asian British Studies. Guest Edited special issue of Interactions I 5.2 (2006): S. I-I68. 
Aufsätze:

- "What am I, anyhow?": Ethnic Consciousness and the Borderlands-Within in Maxine Hong Kingston's and Rebecca Walker's Autobiographies, in: Close Encounters of an Other Kind: New Perspectives on Race, Ethnicity and American Studies, hrsg. von Roy Goldblatt u.a. Joensuu, Finland 2005, S. 3-17.

- "Mei Ng." Voices from the Gaps: Women Artists and Writers of Color. <http://voices. cla.umn.edu/vg/Bios/entries/ng_mei.html>

- Fighting for the Mother/Land: An Ecofeminist Reading of Linda Hogan's "Solar Storms", in: SAIL: Studies in American Indian Literature I7.3 (2005), S. 67-84. reprinted in Contemporary Literary Criticism 265 , hrsg. von Jefr Hunter, Detroit, MI 2009, S. 193-200.

- Motherhood and Mothering as Site of Differences in Barbara Kingsolver's "Pigs in Heaven", in: Journal of the Association for Research on Mothering 8.I+2 (2006), S. 223-232.

- Introduction. "Against the (Main)stream: New Perspectives on Asian American and Asian British Studies", in: Interactions I 5.2 (2006), S. I-I 2.

- Hooked on the American Dream?: Transnational Sexual Labor in Nora Okja Keller's "Fox Girl", in: Feminist Studies in English I 5.2 (2007), S. I 59-I 84.

- Writing Against The Grain: The Cross-Over Genres of Maxine Hong Kingston's "The Woman Warrior", "China Men", and "The Fifth Book of Peace", in: Interactions I6.2 (2007), S. I I I-I 22.

- Writing Rape, Trauma, and Transnationality onto the Female Body: Matrilineal Embody-ment in Nora Okja Keller's "Comfort Woman”, in: Meridians: Feminism, Race, Transnationalism 7.2 (2007), S. 7I-IOO.

- Rewriting American Democracy: Language and Cultural (Dis)Locations in Esmeralda Santiago and Julia Álvarez, in: The Bilingual Review/ La Revista Bilingüe 28. I (Jan-Apr 2004-07), S. 3-15.

- Mother-Daughter Relationships in American Literature, in: Teaching Motherhood: A Collection of Post-Secondary Courses on Mothering/Motherhood, hrsg. von The Association for Research on Mothering (ARM), Toronto, October 2008, S. I97-I 98.

- (Breaking out of) The 'Literary Ghetto': Where to Place Asian American Writers, Or: De-Essentializing Canonicity, in: Hungarian Journal of English and American Studies I 4.2 (2008), S. 287-302.

- Language, Body, Transnational Solidarity: Corporeality in Asian American MotherDaughter Writing, in: Textes et Genres: L'ecriture du corps dans la litterature feminine de langue anglais II, hrsg. von Claire Bazin and Marie-Claude Perrin-Chenour, Paris: Université Paris X, Nanterre 2008, S. 457-468.

- Teaching Kingston's "No Name Woman” as a Primer for Reading Multi-Ethnic American Literature, in: Exploring Spaces: Practices and Perspectives, hrsg. von DorotheA Steiner und Sabine Danner, Münster 2009, S. 53-73.

- Perspectival Adjustments and Hyper-Reality in I I'09"or, in: Invited submission for From Solidarity to Schisms: 9/I I and After in Fiction and Film from Outside the US, hrsg. von Cara Cilano, New York u. Amsterdam 2009, S. I 83-200.

- Art Imitating Life?: Visual Turns in 9/ I I Novels, in: Zeitschrift für Anglistik und Amerikanistik Special Issue on 9/I I, 58.1 (2010), S. 39-54.

- Of Princesses Pari and Fox Girls: Nora Okja Keller's Transnational Performance of Korean Histories and Myths, in: Beyond Culture: Transnational Asian American Warrior 
Women, hrsg. von Lan Dong, Jefferson, NC 20I0, S. 9-25. Reprinted in Contemporary Literary Criticism 340, hrsg. von Jeff Hunter, Detroit, MI 2013, S. 7I-80.

- Trading Spaces, Locating Identities, Writing Selfhood: Lisa Suhair Majaj's and Shirley Geok-lin Lim's Transnational Life-Writing, in: A Fluid Sense of Self: The Politics of Transnational Identity in Anglophone Literatures (= Contributions to Transnational Feminism 3), hrsg. von Silvia Schultermandl und Sebnem Toplu, Wien 20io, S. 25-4I.

- Introduction: A Fluid Sense of Self: The Politics Transnational Identity, in: A Fluid Sense of Self: The Politics of Transnational Identity in Anglophone Literatures (= Contributions to Transnational Feminism 3) hrsg. von Silvia Schultermandl und Sebnem Toplu, Wien 2010, S. 9-22.

- (Zus. mit May Friedman:) Introduction: Growing Up Transnational: Family and Kinship in a Global Era, in: Growing up Transnational: Identity and Kinship in a Global Era, hrsg von May Friedman und Silvia Schultermande, Toronto 20 i i, S. 3-i 8.

- "I am neither the end of the world/ nor the beginning": The Recovery of the Personal as Political in Lisa Suhair Majaj's Self-Writing, in: Growing up Transnational: Identity and Kinship in a Global Era, hrsg. von May Friedman und Silvia Schultermandl, Toronto 20 II, S. 55-68.

- The Politics of Transnational Memory in Amy Tan's “The Joy Luck Club”, in: Journal of Transnational American Studies 3.2 (20I I), [o. pag.]

- From Drug Mule to Miss America: American Exceptionalism and the Commodification of the 'Other' Woman in Maria Full of Grace, in: The Journal of American Culture 34.3 (20II), S. 275-288.

- "What did any of it have to do with race?": Afro-Asian Encounters as Global Narratives of Migration in Cristina García's "Monkey Hunting", in: Atlantic Studies 8. I (20 I I), S. 93-107.

- Remembering what to Forget: Memory as Transnational Practice in Jamaica Kincaid's "Lucy", in: The Transnationalism of American Culture: Literature, Film, and Music, hrsg. von Rocío G. Davis, New York und London 20 I2, S. 44-56.

- (Zus. mit Petra Eckhard und Klaus Rieser:) Introduction: Localizing Global Phenomena of American Culture through Contact, Space and Representation, in: Localizing Global Phenomena: The Contact Spaces of American Culture, hrsg mit Petra Eckhard und Klaus Rieser (= American Studies in Austria io), Wien 20I 2, S. 7-22.

- Die Endlosschleife, in: Hochschullehre XXL - Grosslehrveranstaltungen im Fokus, hrsg. von Alexandra Dorfer und Doris Pany, Graz 2012, S. i 2 I-I 24.

- Transnational Sensibility in Feminist Theory and Practice, in: Between History and Personal Narrative: East European Women's Stories of Migration in the New Millennium, hrsg. von Maria-Sabina Draga Alexandru, Mădălina Nicolaescu, Helen Smith, Wien 2013, S. 269-283.

- "Imagination is a tricky power", in: Transnationalism and Aesthetic Education in Shirley Geok-lin "Lim's Work", in: Asiatic 8.I (June 20I4), S. 40-54.

- Aestheticizing Absence: Representations of the Twin Towers' Shadow in 9/ I I Literature and Film, in: Interactions 23.I-2 (spring-fall 20I4), S. 23 I-42.

- Writing the Self Beyond the Nation-State: The Transnationalism of Olaudah Equiano's "Interesting Narrative", in: Transnational Dimensions of Literature and the Arts. Special Issue of University of Bucharest Review 4.I (2014), S. 32-45.

- The Politics of Transnational Identity in and of Maxine Hong Kingston's "The Woman 
Warrior", in: On the Legacy of Maxine Hong Kingston: The Mulhouse Book, hrsg. von Sämi Ludwig und Nicoleta Alexoae Zagni, Berlin 20 I4, S. 33-44.

- Transnationale Familien als Ort fluider Identitäten, in: Spannungsfeld "Gesellschaftliche Vielfalt": Begegnungen zwischen Wissenschaft und Praxis, hrsg. von Katharina Scherke, Bielefeld 2015, S. I 55-I70.

- Of Literary Letters and IMs: American Epistolary Fiction as Regulative Fictions, in: Thematic chapter for Click and Kin: Transnational Identity and Quick Media, hrsg. von May Friedman und Silvia Schultermandl, Toronto 20i6, S. i i 8-i 36.

- (Zus. mit May Friedman:) Introduction, in: Click and Kin: Transnational Identity and Quick Media, hrsg. von May Friedman und Silvia Schultermande, Toronto 20i6, S. 3-24.

- Nature and the Oppressed Female Body in Nora Okja Keller's "Ecofeminist Aesthetics", in: Ecocriticism and Geocriticism: Overlapping Territories in Environmental and Spatial Literary Studies, hrsg. von Christine M. Battista und Robert J Tally, Jr, New York 2016, S. I7I-I 88.

- Out of Line: Shifting Border Paradigms in Cooper, Morrison and Yamashita, in: Crossing Borders: Essays on Literature, Culture and Society in Honor of Amritjit Singh, hrsg. von Tapan. K. Basu und Tasneem Shahnaaz, Madison, NJ 2017, S. 3-i6.

- Stuplimity and Quick Media Epistolarity in Lauren Myracle's "Internet Girls" Series, in: The Epistolary Renaissance: A Critical Approach to Contemporary Letter Narratives in Anglophone Fiction, hrsg. von Maria Loeschnigg und Rebekka Schum, Berlin 20i8, S. $225-240$.

- Auto Assemblages in SNSs: Intermediality and Transnational Kinship in Online Academic Life Writing, in: Intermediality, Life Writing, and American Studies: Interdisciplinary Approaches, hrsg. von Nassim Winnie Balestrini und Ina Bergman, Berlin 20I8, S. I9I-2IO.

- Reading for Connectivity: Aesthetics and Affect in Intermedial Autobiographies 2.0, in: Autobiography 2.0 and Quick Media Life Writing. special issue of Interactions: Studies in Communications and Culture 9.2 (2018), S. $25 \mathrm{I}-263$.

- (Zus. mit MAY Friedman:) Introduction: Autobiography 2.0 and Quick Media Life Writing, in: Autobiography 2.0 and Quick Media Life Writing. Special issue of Interactions: Studies in Communications and Culture 9.2 (2018), S. I 43-I 54.

- (Zus. mit Katharina Gerund und Anja Mrak:) The Affective Aesthetics of Transnational Feminism, in: WiN EAAS Women's Network Journal I (2018), S. I-23.

Online:

- (Zus. mit David Hucek:) The Border Makes the Stranger: Hospitality and Mobility Justice in Paul Meschuh's "Boat People" (20I6), in: <http://hostfilm.usal.es/index.php/ boat-people-2/>

- (Zus. mit David Hucek:) Hospitality and the Ethics of Disposability in Christoph Kuschnig's Hatch (20I2), in: <https://hostfilm.usal.es/index.php/hatch-2/>

- (Zus. mit David HuceK:) Fatih Akin's The Edge of Heaven (2007) and the Hostipitality of Engagement, in: <https://hostfilm.usal.es/index.php/edge-of-heaven-2/>

Habilitationsschrift:

States of Ambivalence: Transnationalism as Aesthetic Experience in American Literature from Equiano to 9/I I, Graz, 2018, 292 S. 
Kurzfassung:

The study considers transnationalism as an aesthetic experience prompted by American novels which challenge national narratives and the idea of a complete, static, and singular sense of American identity in various ways. It discusses how American novels from the early Republic to the aftermath of the $9 / \mathrm{I}$ I attacks allow readers to explore a sense of ambivalence about the American nation-state by engaging them into aesthetic experiences which prompt them to feel the confusion and distress of protagonists who cannot make sense of national categories in their own identity quests. The study shows that texts which portray the various notions of hybridity and uneven access to modernity of protagonists who need to come to terms with their own fluid identities against the backdrop of prevalent myths of a solidified national American identity, can induce in the reader an acute awareness of the ambivalences that shape the idea of "an" "American" "identity". It investigates the salient markers of the aesthetics of transnationalism and conjoins the insights of aesthetic theory with those of transnational American Studies to formulate a new perspective on the transnational turn in American literature.

Venia:

Amerikanistik.

Institutszuordnung:

Institut für Amerikanistik an der Karl-Franzens-Universität Graz.

\section{Priv. Doz. Dr. Florin Oprescu}

Geboren: I977

B e r uf: Priv. Doz. am Institut für Romanistik der Universität Wien und Assistenz-Professor an der Universitatea de Vest din Timișoara, Rumänien.

Dissertation:

Der Roman und die Morphologie der Macht. Eine rumänische Fallstudie 270 S. [Siehe Publikationen.]

Publikationen:

Monografien:

- Model şi cataliză in lirica românească modernă (Model and Catalysis in Romanian Modern Lyric), Cluj-Napoca 2007, I63 S.

- (In)actualitatea lui Eminescu. Izomorfismele canonului literar (The In-Actuality of Eminescu. The Isomorphism's of the Literary Canon), Bucureşti 2010, $300 \mathrm{~S}$.

- Romanul românesc și morfologia puterii (The Romanian Novel and the Morphology of Power), Iași 201 8, $266 \mathrm{~S}$.

- Power and Literature. Strategies of Subversiveness in the Romanian Novel (= Mimesis 7I), Boston, Berlin 2018, 270 S.

\section{Herausgaben:}

- (Zus. mit Petrea Lindenbauer, Camil Petrescu, Dumitru Tucan:) Identităţi şi alterități socio-istorice în Uniunea Europeană (Identities and Socio-Historical Alterities in EU), Timişoara 2017. 
- (Zus. mit Petrea Lindenbauer und Michael Metzeltin:) The Culture of (Im)Pudicity. The Romanian Case, Frankfurt/M. 2018.

Aufsätze:

- "Nichita Stănescu şi recuperarea modelului eminescian" (Nichita Stănescu and the Recuperation of the Eminescian Model), in: Studii de literatură română şi comparată, vol. XVI-XVII, Timişoara 200I, S. 253-257.

- Dr. Heinrich von Wlislocki - Despre poporul nomad al rromilor (Imagini din viața rromilor din Transilvania) (Dr. Heinrich von Wlislocki - About the Nomad Rroma People. Images from the Rroma Life of Transilvania), in: Revista de etnologie, vol. X, Timişoara 200I, S. 219-222.

- "Călători şi călătorii la capătul nopții" (Voyagers and Voyages at the End of the Night), in: Studii de literatură română şi comparată, vol. XVIII-XIX, Timişoara 2003, S. I79-I 87.

- Les experiences théâtrales modernes, ou «vivre» le théâtre, in: Caiet de semiotică, nr. I4, Timişoara 2003, S. 8 I-84.

- "Paradoxul blagian al modernității" (The Blagian Paradox of Modernity), in: Meridian Blaga, vol. III, Cluj-Napoca 2003, S. I86-192.

- O ipostază a modelului catalitic blagian in lirica română modernă (A Representation of the Catalytic Blagian Model in Modern Romanian Poetry), in: Meridian Blaga, vol. IV, Cluj-Napoca 2004, S. I87-196.

- 75 H.P. Une mise en signe avant-gardiste, in: Caiet de semiotică, nr. I 5 , Timişoara 2004, S. I69-I74.

- O ipoteză asupra destinului scenic al dramaturgiei lui Blaga (A Hypothesis about the Blaga's Theater Destiny), in: Tomis (August 2004), S. 60-62.

- Metamorfoza authorului (Eugen Ionescu - Rinocerii) (The Author Metamorphosis. Eugen Ionescu - The Rhinos), in: Banat, nr. 4-5, (April-May 2004), Lugoj, S. I 3.

- Paşol na turbinca, in Tomis (June 2005), S. 28-30.

- Intrarea prin oglindă (Through the Mirror), in: Tomis (Februar 2005), S. 32-35.

- Modernitatea şi reprezentările lirice blagiene (Modernity and Lyrical Representations of Blaga), in: Magistri honorem. G. I. TohănEAnu, Timişoara 2005, S. 391-402.

- Dicționar al Scriitorilor din Banat (Dictionary of Banat Writers), hrsg von AlexanDRU RUJA (CNCSIS grant), EUVT, Timişoara, 2005. (Articles: "Arieşan, T. Claudiu", "Dima, Simona Grazia" S. 243-246, "Foarță, Şerban" S. 3 I I-3 I 8, "Nemoianu, Virgil" S. 547-554, "Tichindeal, Dimitrie").

- Poetica somnului (The Poetics of Sleep), in: Meridian Blaga. V, Cluj-Napoca 2005, S. I74-I 84 .

- Noul umanism blagian (The New Blagian Humanism), in: Meridian Blaga VI, ClujNapoca 2006, S. I33-I 46.

- Ion Barbu’s "New Humanism", in: Analele Universității de Vest din Timişoara, Seria Filozofie şi Ştiințe ale comunicării, vol. II, XIX (2007), S. 99-I07 and at www.filocom. uvt.ro.

- Procesualitate şi lirism (Procesuality and Lirism), in: Analele Universității de Vest, seria Ştiințe Filologice, Timişoara, XLV (2007), S. 243-248.

- Ipostaze lirice ale Logosului (Lyrical Representations of Logos), in: Studii de literatură română şi comparată, vol. XXII, Timişoara 2007, S. I3 I-I 38.

- Reprezentări lirice ale angelicului (Lyrical Representations of the Angelic), in: Studii de Literatură română şi comparată, vol. XXIII, Timişoara 2007, S. 75-90. 
- Femininul ca fatalitate in dramaturgia blagiană (The Feminine as Fatality in the Theatre of Blaga), in: Meridian Blaga VII, Cluj-Napoca 2007, S. 95-I07.

- Poetica influenței (The Poetics of Influence), in: Meridian Blaga VIII, Cluj-Napoca 2008, S. I I 2-I 25.

- Max Blecher şi exercițiul autenticităţii (Max Blecher and the Authenticity Exercise), in: Studii de Literatură română şi comparată, vol. XIX, Timişoara 2008, S. I I I-I 30.

- "Bertolt Brecht şi metamorfoza epică a teatrului. Opera de trei parale" (Bertolt Brecht and the Epical Metamorphosis of Theater. The Threepenny Opera), in: Analele Universității de Vest, seria Ştiințe Filologice, Timişoara, XLVI (2008), S. I 88-I93.

- Vocația prieteniei (The Friendship Vocation), in: Ion Ianoşi. 8o, Ed. Aura Christi and Alexandru Ştefănescu, București 2008, S. I87-194.

- Der Wiener Expressionismus - Bezugspunkt für Lucian Blagas ästhetische Entwicklung, in: Rumänien und Europa. TransversaleBerlin 2009, S. I 47-1 58.

- «L'épuration» d'un écrivain. Le cas de Lucian Blaga, in: Identité et révolte, Editions de l'Institut d'Etudes Balkaniques, Limes 2009, S. I 5 I-I 56.

- Lucian Blaga şi metaforismul ontologic I (Lucian Blaga and the Metaphorical Ontologism I), in: Meridian Blaga ı, Cluj-Napoca 20 Io, S.75-77.

- George Bacovia - un poet neoromantic eminescian? (George Bacovia - a Neo-Romantical Poet?), in: Concepte in mişcare. Studii despre stadiul actual al criticii şi istoriei literare româneşti, (Concepts in Movement. Studies about the Actual Status of the Romanian Critique and History), Foreword by Dan Mănucă, hrsg. von Ofelia Ichim und Şerban Axinte, Bucureşti 20I0, S. 409-4I9.

- Reinterpreting the Philosophical Canon, in “JSRI”, volume 9, no. 27 (winter 2010), S. 337-343.

- Lucian Blaga şi metaforismul ontologic II (Lucian Blaga and the Metaphorical Ontologism II), in: Meridian Blaga I I, Casa Cărții de Ştiință, Cluj-Napoca 20 I I, S. 77-82.

- Relectura eminescianismului (Re-reading Eminescu), in: Per Teresa. Obiettivo Romania. Studi e Ricerche in Ricordo di Teresa Ferro, Udine 2009, S. 40I-409.

- (Zus. mit Monica Oprescu:) Teacher Training in Romania, in: Problems and Prospects in Higher Education, hrsg. von Gregory T. Papanikos und Nicholas Pappas, Atiner 20I0, S. 279-290.

- Dialectica spiritului ştiințific postmodern sau despre o altă postmodernitate (The Dialectic of Postmodern Scientific Spirit or about a Different Postmodernity), in: Studii de literatură română şi comparată, volumul XX, Timişoara 2010, S. 85-92.

- The Evolution of the Romanian Literary Textbooks and the Transformation of Literary Canon, in: Journal of Educational Science B+, An XIII/ nr. I (20 I I), S. I9-24.

- Literatură și canon istoric (Literature and Historical Canon), in: Philologica Jassyensia, An VII, Nr. 2, I 4 (20II), S. 343-35 I.

- Țiganiada-un canon littéraire manqué, in: Perspectives contemporaines sur le monde medieval, Tiparg, Piteşti 20I I, S. 89-92.

- (Zus. mit Monica Oprescu:) Alternative Methods in Teaching Literature. Transforming the Literary Canon, in: Romanian Journal of English Studies, vol. 8, Timişoara 20 I I, S. I 37-I 45.

- Le masculin confessant et l'Histoire chez Marguerite Yourcenar et Vintilă Horia, in: Marguerite Yourcenar et la culture du masculin, sous la direction de Marc-Jean Filaire, Nimes 201 I , S. 65-75.

- (Zus. mit Monica Oprescu:) A new cultural canon in a new multicultural world: Identity, diversity and multiculturalism between the past and future in Romania's Banat 
region, in: Proceedings of the thirteenth Conference of the Children's Identity and Citizenship in Europe Erasmus Academic Network, Dublin 20 I I, London Metropolitan University, S. 227-233.

- (Zus. mit Monica Oprescu:)Multiple Intelligences in Teaching Literature, in: Romanian Journal of English Studies, nb. 9, Timişoara 201 2, S. I 25 -I 34.

- (Zus. mit Dana Crăciun, Monica Oprescu:) Pre-service teacher training of Romanian students in English and Physics from inter-, pluri-, transdisciplinari perspective, in: Journal of Educational Science and Psychology, Vol III, nr. 2 (2013), S. I-I 3.

- E. M. Cioran and the Self-Image of the Modern Philosopher in the Broken Mirror, in: Procedia - Social and Behavioral Sciences, vol.7I (2013), S. I 82-I88.

- The New Millenium and the Age of Terror. Literature and the Figure in the Carpet, in: „JSRI”, Volume I2, No 36 (2013), S. 5 I-7I.

- Mecanismele canonizării literare. Funcțiile şi disfuncțiile presei de specialitate (The Mechanisms of Literary Canonization. The Functions and the Dysfunctions of Literary Press), in: Rumänien. Medialität und Inszenierung, hrsg. von Maren Huberty, MiChele Mattusch, Valeriu Stancu, Berlin 2013, S. 69-8 I.

- L'avant-garde roumaine et le défi syntétiste, in: Poétiques scientifiques dans les revues européennes de la modernité, des années I900 à I940, hrsg. von TANia Collani und Noelle Cuny, Paris 2013, S. I29-136.

- (Zus. mit Monica Oprescu:) Developing Intercultural Awareness by Integrating Features of Englishness and Romanianness, in: Identities and Citizenship Education: Controversy, crises and challenges, Selected papers from the fifteenth "Conference of the Children's Identity and Citizenship" in Europe Erasmus academic Network, Lisbon 2013 , hrsg. von Peter Cunningham, London 2013, S.I50-I 58.

- Alexandre Soljenitzyne et la mémoire du gouffre, in: Mémoire, Histoire, Témoignage. Essais sur la littérature du goulag Est-Européen, Dir. Dumitru Tucan, Szeged 2014, S. I 5 I-I 68.

- The Anxiety of Cultural Influence. The Exponential Case of Benjamin Fondane, in: Romania in the world. Contacts and reception, hrsg. von Paul NANU, Turku 20 I 4, S. 57-68.

- La poétique du fragmentaire dans les Cahiers d'Emil Cioran, in: L'écriture fragmentale, hrsg. von Pierre Garrigues und Mustapha Trabelsi, Presses de l'Université de Sfax, 2014, S. 76-86.

- (Zus. mit Petrea Lindenbauer:) Geschichtsdiskurs und nationale Identität in Rumänien, in: Wiener Romanistische Landeswissenschaft(en), hrsg von Fabio LongOnI, Wien 2015 , S. II 6-I33.

- Lucian Blaga și soluțiile poeziei populare (Lucian Blaga and the Solutions of Popular Poetry), in: În oglinzile democrației: literatura europeană și etica societară, hrsg. von Tatiana Ciocoi, Emilia Taraburcă, Chişinău 20 i 5, S. 9 I-ioo.

- The Power and Subversiveness of Literature: The Romanian Case, in: Art and Intercultural Dialogue, hrsg. von Susana Gonçalves und Suzanne Majhanovich, Rotterdam, Boston, Taipei 2016, S. 97-133.

- Funcțiile canonului literar și uzul didactic al literaturii (The Functions of Literary Canon and the Didactical Use of Literature), in: Identități și alterități socio-istorice în Uniunea Europeană (Identities and Socio-Historical Alterities in EU), hrsg. von Petrea Lindenbauer, Florin Oprescu, Camil Petrescu, Dumitru Tucan, Vest 20i7, S. 2 i-37.

- (Zus. mit Petrea Lindenbauer:) Imaginarul puterii corpului feminin. Câteva repere istorice În căutarea corpului pierdut, in: Studii interdisciplinare de corpologie feminină, 
(Searching for the Lost Body. Interdisciplinary Studies of Feminin Corpology), hrsg. von Oana Ursache und Paul Nanu, Turku 2017, S. 87-98.

- (Zus. mit Monica Oprescu:) Mateiu Caragiale -Through the Leftovers' Glass. From the Journal to Ephemerides, in: Caietele Echinox, vol. 33 (2017): "Pour une politique du résiduel en littérature", S. I 50-I 59: <http://phantasma.lett.ubbcluj.ro/?page_id=6I 57>

- Les espaces maudits chez Max Blecher et dans le film surréaliste des années 30, in: Räume und Medien in der Romania - Espaces et médias dans les cultures romanes - Spații și medii în culturile romanice, hrsg. von Sabine Krause und Heide Flagner, Hildesheim, Zürich, New York 2018, S. I83-196.

- Das Gedächtnis der Macht - die Macht des Gedächtnisses im rumänischen postkommunistischen Roman, in: Kulturelles Gedächtnis - Ästhetisches Erinnern: Literatur, Film und Kunst in Rumänien, hrsg. von Michèle Mattusch, Berlin 20 I 8, S. 229-249.

\section{Rezensionen:}

- Meridian Blaga V, in: Convorbiri literare, May 2006, S. I45-I 46.

- Ioana Em. Petrescu şi dialectica spiritului ştiințific postmodern (Ioana Em. Petrescu and the Dialectic of Postmodern Scientific Spirit), in: Tribuna, nr. I08, anul VI (I-I 5 Mars 2007), S. 6-7.

- Elanul vitalist de factură dionisiacă (The Vitalist Dionisiac Energy), in: Contemporanul, anul XIX, nr. 2, 67 I (Februar 2008), S. i9.

- Aceasta ar putea fi o pipă (This could be a Pipe), in: Contemporanul, anul XIX, nr.3, 672 (Mars 2008), S. I9.

- Sublimul romantism al avangardei (The Sublime Romanticism of the Avant-Garde), in: Contemporanul, nr. 5 (2008), S. I4.

- Manevrele de toamnă ale lui Petre Stoica la ceas de primăvară (Manevrele de toamnă of Petre Stoica at Spring Hours), in: Orizont, nr. 4 (2008), S. I 5.

- Intoarcerea la valoare (Returning to Value), in: Contemporanul, nr. I I (2009), S. 24.

- Despre treptele cunoaşterii la Virgil Nemoianu (The Steps of Knowledge at Virgil Nemoianu), in: Contemporanul, nr. I2 (2009), S. I6.

- Sodoma şi Gomora, in: Contemporanul, nr. I (2010), S. 34.

- Sublima lecție despre cub (The Sublime Lesson about the Cube), in: Contemporanul, nr. 3 (2010), S. I7.

- Canon şi canonizări in spațiul cultural românesc actual (Canon and Canonization in the Romanian Actual Cultural Space), Contemporanul, nr. 3 (20 I I), S. 27.

- Elogiul textocentrismului și utilitatea teoriei (The Praise of the Textocentrism and the Utility of Theory), Orizont, numărul I I (20I4), S. 22.

- Și acei nebuni, rătăcitori, tăcuți (And those Wandering, Silent Fouls), Orizont, nr. 6 (20I5), S. 23.

- Despre oameni și case sau vârstele imperfecte ale Inocenței, in: Revista 22 (I I/7/2017).

Habilitationsschrift :

Der Roman und die Morphologie der Macht. Eine rumänische Fallstudie. [Siehe Publikationen.]

Kurzfassung:

In der vorliegenden Arbeit >Der Roman und die Morphologie der Macht. Eine rumänische Fallstudier beschäftige ich mich theoretisch und angewandt mit der Art, in der uns die Lite- 
ratur, und insbesondere der Roman, ein Erkenntnisinstrument für die Beziehungen bietet, die zu einem bestimmten Zeitpunkt die Strukturen einer Gesellschaft definieren. Dabei gehe ich von der Voraussetzung aus, dass die Literatur in einer Gesellschaft wie der rumänischen zur Zeit des Kommunismus, die den Machtexzess erfahren hat, oft eine Form der Subversion und Verurteilung der Exzesse war. - Davon ausgehend erforsche ich die Beziehung zwischen der sozialen Macht und dem Roman. Im ersten Teil der Arbeit werden die sozio-politischen und philosophischen Theorien von F. Nietzsche bis M. Weber und die neueren Auffassungen von S. Lukes, S. Parsons, P. Bourdieu und M. Foucault, die wesentlich zur Bestimmung des Begriffs der Macht beigetragen haben, kritisch durchleuchtet. Anschließend wird eine für die narratologische Textanalyse geeignete Typologie der Macht entworfen, welche auch die Zusammenhänge beschreibt, die zwischen den Aktanten in den verschiedenen Handlungsumständen entstehen. - Der zweite Teil der Arbeit ist den Fallstudien gewidmet, die die oben angeführte Typologie anhand von vier repräsentativen Romanen der rumänischen Literatur aus verschiedenen historischen Epochen veranschaulichen soll. Die Analyse der ausgewählten Romane hat einen exemplarischen Charakter für die im ersten Teil erarbeitete Typologie der Macht, aber auch für die Romane der rumänischen Literatur im Allgemeinen.

Venia:

Romanische Literatur.

Institutszuordnung:

Institut für Romanistik der Universität Wien. 
